\title{
Development and Validation of a Seizure Prediction Model in Neonates Following Cardiac Surgery
}

Running Head: Neonatal Seizure Prediction Model

Maryam Y. Naim, M.D., M.S.C.E. ${ }^{1}$, Mary Putt, PhD $^{2}$, Nicholas S. Abend, M.D., M.S.C.E, ${ }^{3}$ Christopher W. Mastropietro, M.D. ${ }^{4}$, Deborah U. Frank, M.D. ${ }^{5}$, Jonathan M. Chen, M.D. ${ }^{6}$, Stephanie Fuller, M.D. ${ }^{6}$, James J. Gangemi, M.D. ${ }^{7}$, J. William Gaynor, M.D. ${ }^{6}$, Kristin Heinan, M.D. ${ }^{8}$, Daniel J. Licht, M.D. ${ }^{3}$, Christopher E. Mascio, M.D. ${ }^{6}$, Shavonne Massey M.D., M.S.C.E. ${ }^{3}$, Mark E. Roeser, M.D. ${ }^{7}$, Clyde J. Smith, M.D. ${ }^{5}$, Stephen E. Kimmel M.D., M.S.C.E. ${ }^{2}$

${ }^{1}$ Division of Cardiac Critical Care Medicine, Departments of Anesthesiology, Critical Care Medicine and Pediatrics, Children's Hospital of Philadelphia (CHOP), University of Pennsylvania Perelman School of Medicine

${ }^{2}$ Department of Biostatistics and Epidemiology, University of Pennsylvania Perelman School of Medicine

${ }^{3}$ Division of Neurology, Departments of Neurology and Pediatrics, CHOP, University of Pennsylvania Perelman School of Medicine

${ }^{4}$ Division of Critical Care, Department of Pediatrics, Riley Hospital for Children at Indiana University Health, Indiana University School of Medicine

${ }^{5}$ Division of Critical Care, Department of Pediatrics, University of Virginia (UVA)

${ }^{6}$ Division of Cardiothoracic Surgery, Department of Surgery, CHOP, University of Pennsylvania. Perelman School of Medicine

${ }^{7}$ Division of Cardiothoracic Surgery, Department of Surgery, UVA

${ }^{8}$ Division of Neurology, Department of Pediatrics, UVA

Funding Statement: Supported by the Endowed Chair of Cardiac Critical Care Medicine at $\mathrm{CHOP}$

Presented at the Society of Thoracic Surgeons $56^{\text {th }}$ Annual Meeting January 2020

Word count: 4509

Abstract: 244

Corresponding Author:

Maryam Y. Naim, M.D

Division of Cardiac Critical Care Medicine

Department of Anesthesiology, Critical Care Medicine and Pediatrics

CHOP

3401 Civic Center Blvd

Philadelphia, PA 19104

Email: naim@email.chop.edu

This is the author's manuscript of the article published in final edited form as:

Naim, M. Y., Putt, M., Abend, N. S., Mastropietro, C. W., Frank, D. U., Chen, J. M., Fuller, S., Gangemi, J. J., Gaynor, J. W., Heinan, K., Licht, D. J., Mascio, C. E., Massey, S., Roeser, M. E., Smith, C. J., \& Kimmel, S. E. (2020). Development and Validation of a Seizure Prediction Model in Neonates Following Cardiac Surgery. The Annals of Thoracic Surgery. https://doi.org/10.1016/j.athoracsur.2020.05.157 


\section{ABSTRACT}

BACKGROUND: Electroencephalographic seizures (ES) following neonatal cardiac surgery are often subclinical and have been associated with poor outcomes. An accurate ES prediction model could allow targeted continuous electroencephalographic monitoring (CEEG) for high-risk neonates.

METHODS: Development and validation of ES prediction models in a multicenter prospective cohort where all postoperative neonates with cardiopulmonary bypass (CPB) underwent CEEG.

RESULTS: ES occurred in $7.4 \%$ of neonates (78 of 1053). Model predictors included gestational age, head circumference, single ventricle defect, DHCA duration, cardiac arrest, nitric oxide, ECMO, and delayed sternal closure. The model performed well in the derivation cohort (c-statistic 0.77 , Hosmer-Lemeshow $\mathrm{p}=0.56$ ), with a net benefit (NB) over monitoring all and none over a threshold probability of $2 \%$ in decision curve analysis (DCA). The model had good calibration in the validation cohort (Hosmer-Lemeshow, $p=0.60$ ); however, discrimination was poor (c-statistic 0.61) and in DCA there was no NB of the prediction model between the threshold probabilities of $8 \%$ and $18 \%$. Using a cut-point that emphasized negative predictive value (NPV) in the derivation cohort, 32\% (236 of 737) of neonates would not undergo CEEG, including 3.5\% (2 of 58) with ES (NPV 99\%, sensitivity 97\%).

CONCLUSIONS: In this large prospective cohort, a prediction model of ES in neonates following CPB had good performance in the derivation cohort with a NB in DCA. However, performance in the validation cohort was weak with poor discrimination, calibration, and no NB in DCA. These findings support CEEG monitoring of all neonates following CPB. 
Continuous electroencephalographic monitoring (CEEG) has identified postoperative electroencephalographic seizures (ES) in neonates following surgery with cardiopulmonary bypass (CPB) which are usually subclinical. ${ }^{1-7}$ Postoperative ES are associated with worse neurodevelopmental outcomes, abnormal neuroimaging and neurologic examinations. ${ }^{1,8-11}$ Based on these data, an American Clinical Neurophysiology Society's (ACNS) guideline recommends performing CEEG following neonatal cardiac surgery. ${ }^{12}$ However, CEEG is resource intense so widespread implementation has not occurred. ${ }^{13}$ Targeting CEEG to neonates at high risk of ES would enable more efficient use of CEEG resources. The aim of this investigation was to develop and validate a prediction model for ES with the hypothesis that preoperative and operative characteristics would predict the probability of postoperative ES.

\section{PATIENTS AND METHODS}

\section{Derivation and validation cohorts}

Models were developed and validated in a prospectively acquired cohort from three centers: Children's Hospital of Philadelphia (CHOP), Riley Children's Hospital $(\mathrm{RCH})$, and University of Virginia Children's Hospital (UVA), where postoperative neonates ( $\leq 30$ days, corrected gestational age $\leq 44$ weeks from June 2012 through 2018 at CHOP, May 2014 through 2018 at $\mathrm{RCH}$, and May 2016 through 2018 at UVA) underwent CEEG. The cohort was identified through the Society of Thoracic Surgeon's database for the individual institution. For multiple operations during the neonatal period, only the index operation was included.

Exclusion criteria were infants $>30$ days, no CPB and no CEEG. Each institutional review board approved the study with waiving informed consent as this was chart review of existing clinical data.

Primary outcome 
The primary outcome ES was defined as an abnormal, paroxysmal EEG event different from the background, $>10$ seconds (or shorter if associated with a clinical seizure), with a plausible EEG field, that evolved in frequency, voltage, morphology and/or spatial distribution within 48 hours postoperatively.

\section{EEG Monitoring}

Postoperative CEEG was initiated within six hours of returning to the intensive care unit (ICU). CEEG was performed with the international 10-20 system (modified for neonates) using portable acquisition machines networked to the main EEG server, allowing EEG review at the bedside, from multiple sites in the hospital, and remotely. If an ES was identified, the ICU Service was alerted by the EEG technologist or electroencephalographer, and neurology consultation was obtained. CEEG was continued on all neonates for 48 hours. In those with ES, CEEG was continued for 24 hours following the termination of the last ES. Antiseizure medication choice was at the discretion of the treating cardiac intensivist and neurologist.

\section{Data collection}

Data were collected and managed using REDCap (Research Electronic Data Capture), hosted at the CHOP Research Institute. ${ }^{14}$ Clinical data were obtained from the medical record. Neonates were categorized according to a classification that incorporates cardiac anatomy and perioperative physiology, which has been shown to predict perioperative mortality. ${ }^{15} \mathrm{~A}$ list of operations and comparison across centers is provided in the Supplemental Material (Supplemental Table 1). ES were classified as status epilepticus if any ES lasted longer than 30 minutes or if recurrent ES lasted more than 30 minutes in any one-hour epoch (50\% seizure burden). ES were classified as EEG-only ES (no clinical signs observed by bedside caregivers or on video review) or electroclinical ES. 


\section{Candidate predictors}

Candidate predictors included 23 preoperative and operative variables (Supplemental Table 2). In neonates with CHD seizures, brain injury and neurodevelopmental dysfunction have been associated with age at surgery, sex, race, gestational age, low birth weight, head circumference, genetic syndrome, cardiac defect, duration of deep hypothermic circulatory arrest (DHCA), open sternum, cardiac arrest, low hematocrit on bypass and extracorporeal membrane oxygenation (ECMO)..$^{1,2,4,5,7,11,16-20}$ Racial and ethnic disparities have also been observed with CHD outcomes. ${ }^{21-24}$ Regional cerebral perfusion (RCP) has been proposed as a neuroprotective strategy during CPB to avoid DHCA, although ES have been associated with its use. ${ }^{4,7,25}$ Longer duration of CPB, and inhaled nitric oxide were used as surrogates for a sicker postoperative neonate.

\section{Model building strategy}

Statistical analyses were performed using Stata 14.2 (College Station, TX). The association of each predictor with ES was examined using the chi-square or Fisher's exact test for categorical variables and the Wilcoxon's rank sum test for continuous variables. Significant center variation was observed in preoperative and operative variables (Supplemental Table 3). Therefore, the initial building strategy to derive a model in the CHOP dataset which comprised $70 \%$ (739 of 1053) of the cohort was abandoned. These models had inadequate fit in datasets from $\mathrm{RCH}$ and UVA (Supplemental Tables 4, 5, 6 and 7). To make the model more generalizabile to centers other than those in this study a combined dataset was used to incorporate the variability in neonatal cardiac surgery perioperative care. ${ }^{26-28}$ The cohort from the three centers was combined with a random 70:30 split for derivation and validation of the model, respectively. Variables with a p-value of $<0.2$ (two-sided) were entered into a model using either logistic regression with backward elimination or with Least Absolute Shrinkage and Selection Operator regression (lasso). In backward elimination, variables were sequentially 
removed based on their beta coefficients with comparison of the area under the receiver operating characteristic curve $(A \cup C)$ until the model was deemed adequate. Lasso regression is a machine learning algorithm that both shrinks coefficients of variables towards zero to avoid overfitting and selects a subset of predictor variables into the model based on a penalty on the absolute value of the coefficients in the modelzero. ${ }^{29}$

\section{Model assessment and validation}

In the derivation cohort, internal validation was performed using the following: (1) predictive accuracy was assessed by the Brier score (variation explained by predictor, a Brier score of 0 indicates a perfect model whereas 0.25 indicates a non-informative model); ${ }^{30,31}$ (2) discrimination (ability of the model to separate neonates with ES to those without ES) was assessed using the discrimination slope (difference of average predictions in neonates with and without ES), ${ }^{31}$ sensitivity, specificity, and the AUC with the concordance statistic (c-statistic); (3) calibration (accuracy of predictions) was measured using the Hosmer-Lemeshow goodness of fit test and by a calibration plot that assessed agreement between predicted and observed ES; ${ }^{31}$ and (4) decision curve analysis (DCA) was conducted to measure the net benefit (NB) of making decisions based on model predictions. In DCA, the NB of the prediction model is calculated across a range of threshold probabilities for ES, defined as the minimal probability of ES at which EEG monitoring would be warranted. In DCA the prediction model is compared to the default strategies of monitoring all or none. The interpretation of DCA is that the strategy

with the highest NB at a particular threshold probability has the highest clinical value. ${ }^{31,32}$ Crossvalidation using 10 -folds was performed in lasso with selection of the lasso tuning parameter that minimized the estimate of out of sample prediction error.

The model was then applied to the validation cohort to test its performance using all the measures above. Predicted ES probabilities were calculated from the model and sensitivity, specificity, positive predictive values, negative predictive values (NPV), and the proportion of 
patients undergoing CEEG were assessed at various model cutoffs assuming that those above the cutoff would be selected for CEEG.

\section{RESULTS}

Postoperative CEEG occurred in 739 of 745 (99.2\%) neonates at CHOP, 188 of 188 $(100 \%)$ at $\mathrm{RCH}$ and 126 of $130(96.9 \%)$ at UVA. ES occurred in $7.4 \%$ (78 of 1053$)$ of neonates, including $8 \%$ (59 of 739$)$ at $\mathrm{CHOP}, 9 \%(17$ of 188$)$ at $\mathrm{RCH}$, and $1.6 \%(2$ of 126$)$ at UVA. Table 1 provides summary characteristics in the derivation and validation cohorts. ES occurred in $7.9 \%$ (58 of 737 ) of the derivation cohort and $6.3 \%$ (20 of 316 ) of the validation cohort, $p=0.38$. In the derivation cohort, ES occurred in 8.5\% (55 of 521) from CHOP, $10.4 \%$ (13 of 125) from $\mathrm{RCH}$, and $1.1 \%$ (1 of 91 ) from UVA. In the validation cohort, ES occurred in 6.9\% (15 of 281) from CHOP, $6.4 \%$ (4 of 63 ) from $\mathrm{RCH}$, and $2.9 \%$ (1 of 35) from UVA. The final model (Table 2 ) was developed using the lasso due to its superior performance in the validation cohort (Table 3) when compared to backward elimination (Supplemental Tables 8 and 9). In the derivation cohort, the model performed well with a Brier Score of 0.06 indicating an informative prediction. Model discrimination was fair with the predicted probability of ES $11 \%$ higher in neonates with versus without ES (c-statistic of 0.77) (Figure 1 Upper left). Hosmer-Lemeshow suggested

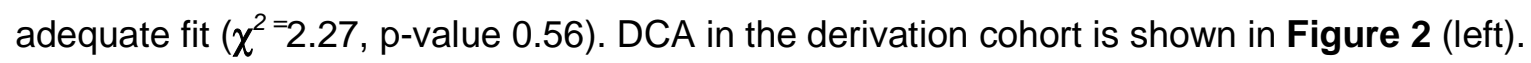
Below the threshold probability of $2 \%$ there was no difference in the NB of monitoring all and monitoring based on the model, with a higher NB for these strategies compared to monitoring none. Above the threshold probability of $2 \%$ the NB of the model was higher than the NB of monitoring all and none.

In the validation cohort the Brier Score was 0.06 indicating an informative prediction. Model discrimination was poor with the predicted probability $6 \%$ higher in neonates with versus those without ES (c-statistic of 0.61) (Figure 1 Upper right). Hosmer-Lemeshow suggested adequate fit $\left(\chi^{2}=6.42, p\right.$ value 0.60$)$; however, the calibration plot (Figure 1 Middle right ) 
showed that predicted probabilities of ES underestimated the observed probabilities. There were a number of neonates in whom the model predicted a low probability of ES, but in whom the actual probability was considerably higher. DCA in the validation cohort (Figure 2 right) showed no difference in the strategy of monitoring all and monitoring based on the model below a threshold probability of $5 \%$. Above a threshold probability of ES of $5 \%$ to a probability of $8 \%$ the model had a NB over monitoring all and none. Above this probability the model had no NB until a threshold probability of $18 \%$ above which it was beneficial till a probability of $50 \%$.

Tables $\mathbf{4}$ and 5 provide model characteristics in the derivation and validation cohorts at different cutoffs. At a cutoff of 0.03 that maximizes sensitivity and NPV, 32\% (236 of 737 ) of neonates in the derivation cohort would not undergo CEEG, including $3.5 \%$ (2 of 58 ) of neonates with ES (NPV 99\%, sensitivity $97 \%$ ). In the validation cohort $25.6 \%$ (81 of 316 ) of neonates would not undergo CEEG, including 10\% (2 of 20) of neonates with ES (NPV 97.5\%, sensitivity $90 \%)$.

\section{COMMENT}

A predictive model based on clinical characteristics was developed and validated to enable efficient use of resources to target CEEG to neonates with a high probability of ES. In the derivation cohort the model overall had good performance with fair discrimination and good calibration. In the validation cohort the model was informative, however discrimination was poor, and although the calibration appeared adequate, the predicted probabilities underestimated the observed probabilities, particularly for neonates with low predicted probabilities. In DCA, the model had some NB in the validation cohort; however, there was no benefit observed between threshold probabilities of $8 \%$ to $18 \%$. Given that the reported probability of postoperative ES is $1.5 \%$ to $20 \%$ and $>8 \%$ in most reports, centers with abundant CEEG resources would likely monitor neonates above the threshold probability of $8 \%$ to $10 \% \cdot{ }^{1-7}$ Given that in the validation cohort the model had no NB in this range it is not of clinical value in centers with abundant 
CEEG resources. ${ }^{17,33,34}$ In centers with limited CEEG resources where higher threshold probabilities for seizures may be used e.g. $50 \%$ the model again had no NB in the validation cohort therefore would not be of clinical value. Therefore, although this model performed well in the derivation cohort, validation was poor indicating the model should not be used for targeting CEEG. These data support the current recommended practice of performing CEEG for all neonates following surgery with $\mathrm{CPB} .^{12}$

Despite the current ACNS guideline, the data on the incidence of postoperative ES, and the association of ES with worse outcomes, CEEG in postoperative neonates has not been widely implemented given the resource requirements. ${ }^{1-12,17}$ Many centers monitor neonates considered to be at the highest risk of ES, including those on ECMO support or following a cardiac arrest. ${ }^{35,36}$ Therefore, a prediction model could allow targeted CEEG. However, although there was a similar ES incidence in 2 of 3 the centers, significant center variation was observed in the timing of surgery, the use of open sternum, intraoperative strategies for neuroprotection including DHCA and RCP, and other factors associated with neurological injury including the lowest hematocrit on CPB, cardiac arrest, and ECMO. The model was therefore developed in a multicenter cohort to enable generalizability.

There are limitations to these data. First, exclusion of CEEG background may have led to information bias. As the goal of this study was to develop a prediction model based on preoperative and operative variables, background CEEG variables were not included. Initial CEEG background has been shown to be useful when incorporated into an ES prediction model in children admitted with acute encephalopathy to the pediatric ICU ${ }^{37}$ Second, perioperative brain injury evident on neuroimaging is an important variable that was not included in the prediction model as all neonates did not have routine perioperative neuroimaging. Third, given the multicenter nature of study misclassification of ES and differences in EEG interpretation may have occurred. A standard definition of ES and ES identification was used which is known to be reliable across encephalographers. ${ }^{38,39}$ Fourth, the study was designed to for a sample size of 
750 neonates in the validation cohort with 60 ES to achieve a sensitivity of $98 \%$ (anticipated $95 \% \mathrm{Cl} 92-100 \%$ ) and specificity of $50 \%$ (anticipated $95 \% \mathrm{Cl}$ of $47-53 \%$ ). The total sample size was achieved, and the number of ES (57) approached the target value. However, in the derivation dataset, while a sensitivity of $97 \%$ was obtained for the lower cutoff of the receiver operating characteristics curve (Table 4), the specificity was only $34.5 \%$, well outside the specified range, and which drove down the NPV. Alternatively, in order to achieve a specificity near $50 \%$, the sensitivity dropped to $85 \%$. This suggests that key predictors needed to achieve these levels of accuracy were missing even from the derivation model. Not surprisingly this resulted in poor performance in the validation cohort.

In conclusion, ES in neonates following surgery with CPB were not accurately predicted by a model using preoperative and operative variables. These findings support continued CEEG in all neonates following surgery with CPB. 


\section{References}

1. Gaynor JW, Jarvik GP, Bernbaum J, et al. The relationship of postoperative electrographic seizures to neurodevelopmental outcome at 1 year of age after neonatal and infant cardiac surgery. J Thorac Cardiovasc Surg. 2006;131(1):181-189.

2. Gaynor JW, Nicolson SC, Jarvik GP, et al. Increasing duration of deep hypothermic circulatory arrest is associated with an increased incidence of postoperative electroencephalographic seizures. J Thorac Cardiovasc Surg. 2005;130(5):1278-1286.

3. Clancy RR, Sharif U, Ichord R, et al. Electrographic neonatal seizures after infant heart surgery. Epilepsia. 2005;46(1):84-90.

4. Newburger JW, Jonas RA, Wernovsky G, et al. A comparison of the perioperative neurologic effects of hypothermic circulatory arrest versus low-flow cardiopulmonary bypass in infant heart surgery. N Engl J Med. 1993;329(15):1057-1064.

5. Helmers SL, Wypij D, Constantinou JE, et al. Perioperative electroencephalographic seizures in infants undergoing repair of complex congenital cardiac defects. Electroencephalogr Clin Neurophysiol. 1997;102(1):27-36.

6. Andropoulos DB, Mizrahi EM, Hrachovy RA, et al. Electroencephalographic seizures after neonatal cardiac surgery with high-flow cardiopulmonary bypass. Anesth Analg. 2010;110(6):1680-1685.

7. Gunn JK, Beca J, Hunt RW, Olischar M, Shekerdemian LS. Perioperative amplitudeintegrated EEG and neurodevelopment in infants with congenital heart disease. Intensive Care Med. 2012;38(9):1539-1547.

8. Bellinger DC, Jonas RA, Rappaport LA, et al. Developmental and neurologic status of children after heart surgery with hypothermic circulatory arrest or low-flow cardiopulmonary bypass. N Engl J Med. 1995;332(9):549-555.

9. Rappaport LA, Wypij D, Bellinger DC, et al. Relation of seizures after cardiac surgery in early infancy to neurodevelopmental outcome. Boston Circulatory Arrest Study Group. Circulation. 1998;97(8):773-779.

10. Bellinger DC, Wypij D, Kuban KC, et al. Developmental and neurological status of children at 4 years of age after heart surgery with hypothermic circulatory arrest or lowflow cardiopulmonary bypass. Circulation. 1999;100(5):526-532.

11. Bellinger DC, Wypij D, Rivkin MJ, et al. Adolescents with d-transposition of the great arteries corrected with the arterial switch procedure: neuropsychological assessment and structural brain imaging. Circulation. 2011;124(12):1361-1369.

12. Shellhaas RA, Chang T, Tsuchida T, et al. The American Clinical Neurophysiology Society's Guideline on Continuous Electroencephalography Monitoring in Neonates. $J$ Clin Neurophysiol. 2011;28(6):611-617.

13. Gutierrez-Colina AM, Topjian AA, Dlugos DJ, Abend NS. Electroencephalogram monitoring in critically ill children: indications and strategies. Pediatric neurology. 2012;46(3):158-161.

14. Wechsler LR, Tsao JW, Levine SR, et al. Teleneurology applications: Report of the Telemedicine Work Group of the American Academy of Neurology. Neurology. 2013;80(7):670-676.

15. Clancy RR, McGaurn SA, Wernovsky G, et al. Preoperative risk-of-death prediction model in heart surgery with deep hypothermic circulatory arrest in the neonate. $J$ Thorac Cardiovasc Surg. 2000;119(2):347-357.

16. Jonas RA, Wypij D, Roth SJ, et al. The influence of hemodilution on outcome after hypothermic cardiopulmonary bypass: results of a randomized trial in infants. $J$ Thorac Cardiovasc Surg. 2003;126(6):1765-1774. 
17. Naim MY, Gaynor JW, Chen J, et al. Subclinical seizures identified by postoperative electroencephalographic monitoring are common after neonatal cardiac surgery. $J$ Thorac Cardiovasc Surg. 2015;150(1):169-178; discussion 178-180.

18. Williams IA, Fifer WP, Andrews H. Fetal Growth and Neurodevelopmental Outcome in Congenital Heart Disease. Pediatr Cardiol. 2015;36(6):1135-1144.

19. Gaynor JW, Stopp C, Wypij D, et al. Neurodevelopmental outcomes after cardiac surgery in infancy. Pediatrics. 2015;135(5):816-825.

20. International Cardiac Collaborative on Neurodevelopment I. Impact of Operative and Postoperative Factors on Neurodevelopmental Outcomes After Cardiac Operations. Ann Thorac Surg. 2016;102(3):843-849.

21. Peyvandi S, Baer RJ, Moon-Grady AJ, et al. Socioeconomic Mediators of Racial and Ethnic Disparities in Congenital Heart Disease Outcomes: A Population-Based Study in California. J Am Heart Assoc. 2018;7(20):e010342.

22. Oster ME, Strickland MJ, Mahle WT. Racial and ethnic disparities in post-operative mortality following congenital heart surgery. J Pediatr. 2011;159(2):222-226.

23. Nembhard WN, Salemi JL, Ethen MK, Fixler DE, Dimaggio A, Canfield MA. Racial/Ethnic disparities in risk of early childhood mortality among children with congenital heart defects. Pediatrics. 2011;127(5):e1128-1138.

24. Wang Y, Liu G, Druschel CM, Kirby RS. Maternal race/ethnicity and survival experience of children with congenital heart disease. J Pediatr. 2013;163(5):1437-1442 e1431-1432.

25. Pigula FA, Nemoto EM, Griffith BP, Siewers RD. Regional low-flow perfusion provides cerebral circulatory support during neonatal aortic arch reconstruction. J Thorac Cardiovasc Surg. 2000;119(2):331-339.

26. Meyer DB, Jacobs JP, Hill K, Wallace AS, Bateson B, Jacobs ML. Variation in Perfusion Strategies for Neonatal and Infant Aortic Arch Repair: Contemporary Practice in the STS Congenital Heart Surgery Database. World J Pediatr Congenit Heart Surg. 2016;7(5):638-644.

27. O'Byrne ML, Glatz AC, Song L, et al. Association Between Variation in Preoperative Care Before Arterial Switch Operation and Outcomes in Patients With Transposition of the Great Arteries. Circulation. 2018;138(19):2119-2129.

28. Justice AC, Covinsky KE, Berlin JA. Assessing the generalizability of prognostic information. Ann Intern Med. 1999;130(6):515-524.

29. Tibshirani R. Regression and shinkage via the Lasso. J R Stat Soc Ser B. 1996;58:267288.

30. Gerds TA, Cai T, Schumacher M. The performance of risk prediction models. Biom J. 2008;50(4):457-479.

31. Steyerberg EW, Vickers AJ, Cook NR, et al. Assessing the performance of prediction models: a framework for traditional and novel measures. Epidemiology. 2010;21(1):128138.

32. Vickers AJ, Elkin EB. Decision curve analysis: a novel method for evaluating prediction models. Med Decis Making. 2006;26(6):565-574.

33. Helmers SL, Wypij D, Constantinou JE, et al. Perioperative electroencephalographic seizures in infants undergoing repair of complex congenital cardiac defects.

Electroencephalogr Clin Neurophysiol. 1997;102(1):27-36.

34. Gaynor JW, Jarvik GP, Gerdes M, et al. Postoperative electroencephalographic seizures are associated with deficits in executive function and social behaviors at 4 years of age following cardiac surgery in infancy. J Thorac Cardiovasc Surg. 2013;146(1):132-137.

35. Lin JJ, Banwell BL, Berg RA, et al. Electrographic Seizures in Children and Neonates Undergoing Extracorporeal Membrane Oxygenation. Pediatr Crit Care Med.

2017;18(3):249-257. 
36. Abend NS, Topjian A, Ichord R, et al. Electroencephalographic monitoring during hypothermia after pediatric cardiac arrest. Neurology. 2009;72(22):1931-1940.

37. Yang A, Arndt DH, Berg RA, et al. Development and validation of a seizure prediction model in critically ill children. Seizure. 2015;25:104-111.

38. Abend NS, Gutierrez-Colina A, Zhao H, et al. Interobserver reproducibility of electroencephalogram interpretation in critically ill children. J Clin Neurophysiol. 2011;28(1):15-19.

39. Hirsch LJ, LaRoche SM, Gaspard N, et al. American Clinical Neurophysiology Society's Standardized Critical Care EEG Terminology: 2012 version. J Clin Neurophysiol. 2013;30(1):1-27. 
Table 1. Comparison of variables in the derivation and validation cohorts

\begin{tabular}{|c|c|c|c|}
\hline Variable & $\begin{array}{l}\text { Derivation } \\
(\mathrm{n}=737)\end{array}$ & $\begin{array}{l}\text { Validation } \\
(\mathrm{n}=316)\end{array}$ & $p$ value ${ }^{*}$ \\
\hline Age at surgery median (IQR) & $6(4,9)$ & $6(4,11)$ & 0.27 \\
\hline Male sex $n(\%)$ & $444(60.2)$ & $198(62.7)$ & 0.46 \\
\hline \multicolumn{4}{|l|}{ Race $\mathrm{n}(\%)$} \\
\hline White & $451(61.0)$ & $196(62.0)$ & 0.64 \\
\hline Black & $94(12.7)$ & $35(11.1)$ & \\
\hline Other & $192(26.1)$ & $85(26.9)$ & \\
\hline $\begin{array}{l}\text { Gestational age at birth (weeks) median } \\
\text { (IQR) }\end{array}$ & $39(38,39)$ & $39(37,39)$ & 0.07 \\
\hline Birth weight $(\mathbf{k g})$ median (IQR) & $3.2(2.8,3.5)$ & $3.1(2.8,3.5)$ & 0.07 \\
\hline Head circumference (cm) median (IQR) & $33.5(32,34.5)$ & $33.5(32,34.5)$ & 0.25 \\
\hline Prenatal diagnosis $n(\%)$ & $537(72.8)$ & $233(73.7)$ & 0.77 \\
\hline \multicolumn{4}{|l|}{ Cardiac defect $n(\%)$} \\
\hline Single ventricle & $254(34.5)$ & $102(32.3)$ & 0.49 \\
\hline Two ventricles without arch obstruction & $315(42.7)$ & $150(47.5)$ & 0.16 \\
\hline Two ventricles with arch obstruction & $168(22.8)$ & $64(20.3)$ & 0.36 \\
\hline Single ventricle without arch obstruction & $54(7.3)$ & $24(7.6)$ & 0.88 \\
\hline Single ventricle with arch obstruction & $200(27.1)$ & $78(24.7)$ & 0.41 \\
\hline CPB duration (minutes) median (IQR) & $60(42,109)$ & $60(41,101)$ & 0.47 \\
\hline DHCA n (\%) & $355(48.2)$ & $145(45.9)$ & 0.50 \\
\hline DHCA duration (minutes) median (IQR) & $37(24,47)$ & $40(25,47)$ & 0.30 \\
\hline RCP n (\%) & $103(14.0)$ & $34(10.8)$ & 0.16 \\
\hline RCP duration (minutes) median (IQR) & $62(39,98)$ & $52(29,72)$ & 0.09 \\
\hline $\begin{array}{l}\text { Lowest hematocrit on CPB (\%) median } \\
\text { (IQR) }\end{array}$ & $28(26,31)$ & $28(26,31)$ & 0.89 \\
\hline Nitric oxide in OR n (\%) & $153(20.8)$ & $64(20.3)$ & 0.85 \\
\hline Cardiac arrest in OR n (\%) & $6(1)$ & $1(0)$ & 0.36 \\
\hline ECMO in OR n (\%) & $30(4)$ & 9 (3) & 0.34 \\
\hline Open chest in OR n (\%) & $205(27.8)$ & $92(29.1)$ & 0.67 \\
\hline Electrographic seizures n (\%) & $58(7.9)$ & $20(6.3)$ & 0.38 \\
\hline
\end{tabular}

Abbreviations: $n$, number; IQR, interquartile; kg, kilogram; cm, centimeters; CPB, cardiopulmonary bypass; DHCA, deep hypothermic circulatory arrest; RCP, regional cerebral perfusion; ECMO, extracorporeal membrane oxygenation; OR, operating room 
Table 2. Prediction model ${ }^{*}$ coefficients

\begin{tabular}{lc}
\hline \multicolumn{1}{c}{ Parameter } & Coefficient \\
\hline Intercept & 6.802 \\
Gestational age (weeks) & -0.117 \\
Head circumference (cm) & -0.181 \\
Single ventricle (yes versus No) & 0.555 \\
DHCA duration (minutes) & 0.018 \\
Nitric oxide in OR (yes versus no) & 0.710 \\
Cardiac arrest in OR (yes versus & 1.645 \\
no) & 1.114 \\
ECMO in OR (yes versus no) & 0.580 \\
Open chest in OR (yes versus no) & "The final prediction model in the derivation dataset was derived from LassoLogit
\end{tabular}


Table 3. Model performance in the derivation and validation cohorts

\begin{tabular}{lcc}
\hline Statistic & Derivation & Validation \\
\hline Likelihood Ratio & 59.99 & \\
Pseudo-R-square & 0.148 & \\
C-statistic & 0.7678 & 0.606 \\
Brier Score & 0.064 & 0.058 \\
Hosmer-Lemeshow $\chi^{2}(p$ value) & $2.27(0.56)$ & $6.42(0.60)$ \\
\hline
\end{tabular}


Table 4. Model characteristics at various cutoffs in derivation dataset

\begin{tabular}{lccccc}
\hline $\begin{array}{l}\text { Model cutoff } \\
\text { Predicted } \\
\text { probability of } \\
\text { ES }\end{array}$ & Sensitivity & Specificity & PPV & NPV & $\begin{array}{l}\text { Proportion } \\
\text { with CEEG }\end{array}$ \\
\hline $\mathbf{0 . 0 2}$ & 96.6 & 15.9 & 8.9 & 98.2 & \\
$\mathbf{0 . 0 3}$ & 96.6 & 34.5 & 11.2 & 99.2 & 65.1 \\
$\mathbf{0 . 0 4}$ & 89.7 & 44.9 & 12.2 & 98.1 & 57.8 \\
$\mathbf{0 . 0 5}$ & 84.5 & 53.8 & 13.5 & 97.6 & 49.3 \\
$\mathbf{0 . 0 6}$ & 74.1 & 61.1 & 14.0 & 96.5 & 41.7 \\
$\mathbf{0 . 0 7}$ & 67.2 & 67.2 & 14.9 & 96.0 & 35.6 \\
$\mathbf{0 . 0 8}$ & 58.6 & 72.5 & 15.4 & 95.3 & 30.0 \\
$\mathbf{0 . 0 9}$ & 55.2 & 76.6 & 16.8 & 95.2 & 25.9 \\
$\mathbf{0 . 1 0}$ & 53.4 & 80.4 & 18.9 & 95.3 & 22.5 \\
$\mathbf{0 . 2 0}$ & 31.0 & 94.7 & 33.3 & 94.1 & 7.3 \\
\hline Abbreviations: ES, electrographic seizures; PPV, positive predictive value; NPV, negative \\
predictive value
\end{tabular}


Table 5. Model characteristics at various cutoffs in validation dataset

\begin{tabular}{lccccc}
\hline $\begin{array}{l}\text { Model cutoff } \\
\text { Predicted } \\
\text { probability of }\end{array}$ & Sensitivity & Specificity & PPV & NPV & $\begin{array}{l}\text { Proportion } \\
\text { with CEEG }\end{array}$ \\
ES & & & & & \\
\hline $\mathbf{0 . 0 2}$ & 95.0 & 11.5 & 6.8 & 97.1 & 88.9 \\
$\mathbf{0 . 0 3}$ & 90.0 & 26.7 & 7.7 & 97.5 & 74.4 \\
$\mathbf{0 . 0 4}$ & 70.0 & 42.2 & 7.6 & 95.4 & 58.5 \\
$\mathbf{0 . 0 5}$ & 65.0 & 50.7 & 8.2 & 95.5 & 50.3 \\
$\mathbf{0 . 0 6}$ & 50.0 & 59.5 & 7.7 & 94.6 & 41.1 \\
$\mathbf{0 . 0 7}$ & 45.0 & 66.2 & 8.3 & 94.7 & 34.5 \\
$\mathbf{0 . 0 8}$ & 40.0 & 71.3 & 8.6 & 94.6 & 29.4 \\
$\mathbf{0 . 0 9}$ & 35.0 & 74.7 & 8.5 & 94.4 & 26.0 \\
$\mathbf{0 . 1 0}$ & 30.0 & 76.4 & 7.9 & 94.2 & 24.1 \\
$\mathbf{0 . 2 0}$ & 20.2 & 95.3 & 22.2 & 94.6 & 5.7 \\
\hline
\end{tabular}




\section{Figure Legends}

Figure 1. Characteristics for the derivation (left) and validation (right) cohorts. Top Receiver operating characteristic curve. Middle Scatter plots of predicted compared to observed probabilities showing model calibration. Bottom Box plots of predicted probabilities showing model discrimination.

Figure 2. Decision curve analysis of the prediction model for the derivation (left) and validation (right) cohorts. 

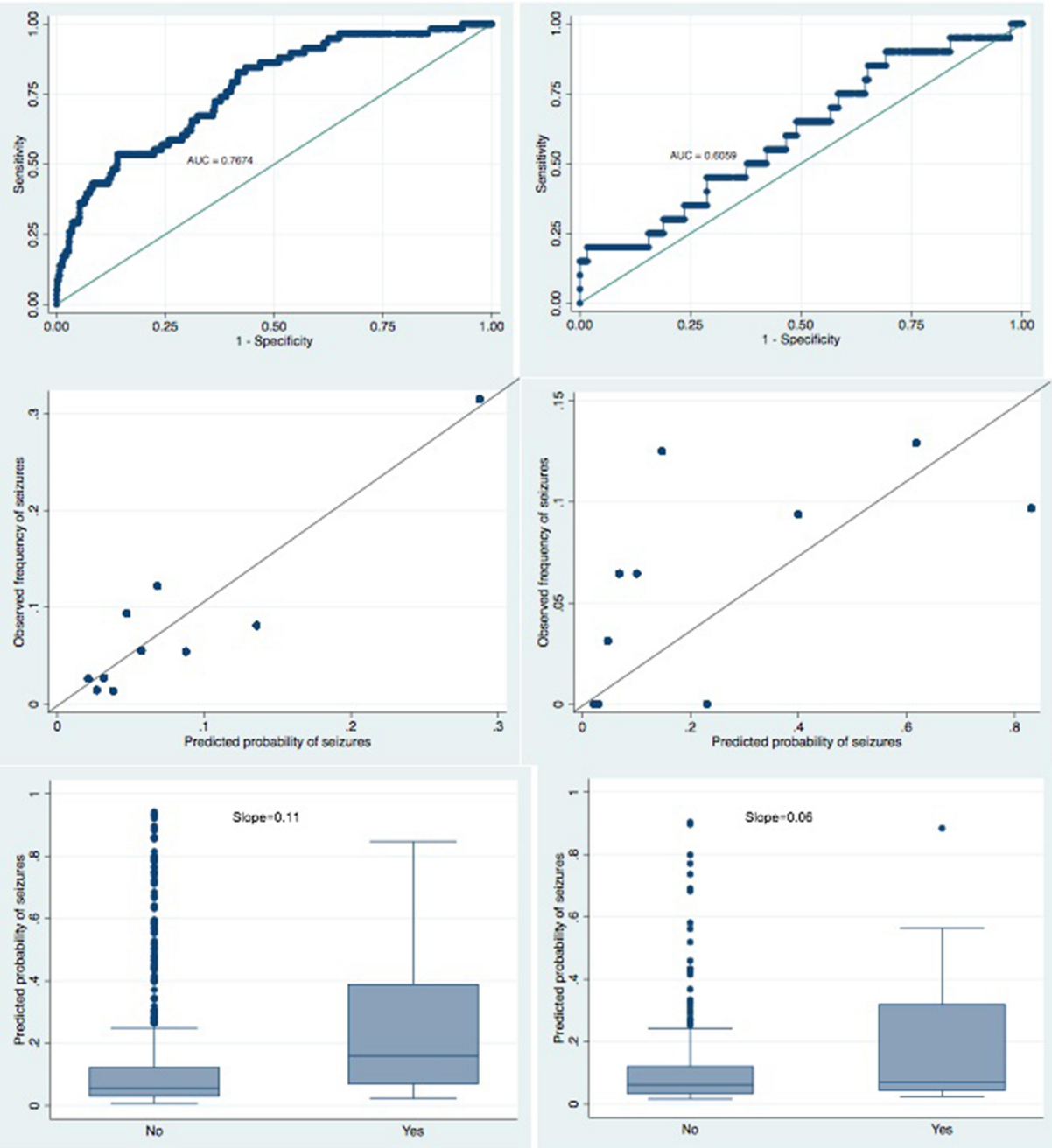

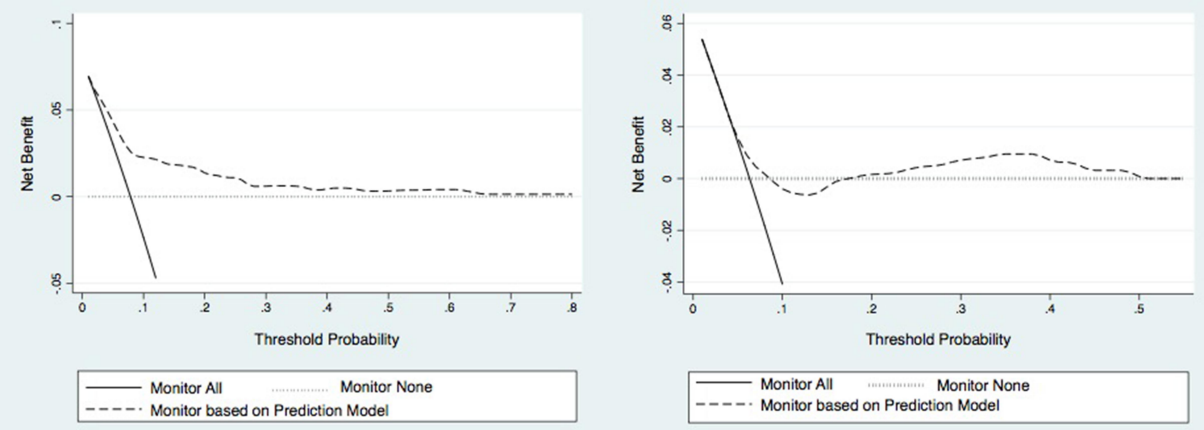